\title{
Regadenoson and exercise myocardial perfusion imaging: The courtship continues
}

\author{
Harkawal S. Hundal, MD, MS, ${ }^{\mathrm{a}, \mathrm{b}}$ and Gregory S. Thomas, MD, MPH, FACC, \\ FASNC $^{\mathrm{a}, \mathrm{b}}$
}

\section{See related article, pp. 336-343}

American Society of Nuclear Cardiology (ASNC) guidelines support the use of low-level exercise testing for adenosine and dipyridamole in select patients to improve diagnostic accuracy and to decrease adverse side effects. ${ }^{1}$ Regadenoson does not have the same support in the current guidelines and, as with the adenosine and diypridamole, its use with exercise remains "off-label", 2 Since approval in 2008, regadenoson has become the most common vasodilator stress test agent in the United States. ${ }^{3}$ Its ease of use as a single bolus injection makes it versatile and straightforward to use. Although approved for supine testing, it has been evaluated for low-level exercise (a protocol we characterize as RegLowEx) in one randomized trial and a large observational study. ${ }^{4,5}$ Similar to adenosine and dipyridamole, the goals of RegLowEx are improved image quality and decreased vasodilator associated symptoms. Newer studies have evaluated regadenoson given just before peak exercise (which we term RegNearPeakEx), at peak (termed RegPeakEx), and just after peak exercise (termed RegPostPeakEx) for those unable to achieve $85 \%$ of maximum predicted heart rate or meet an ischemic endpoint. ${ }^{6-9}$ The primary goal of protocols that allow patients to exercise to peak is to obtain prognostic information from the exercise stress aspect of the test and use regadenoson on an provisional basis such that perfusion imaging sensitivity is not

From the MemorialCare Heart \& Vascular Center, ${ }^{\text {a }}$ Long Beach Memorial Medical Center and Miller Childrenós Hospital of Long Beach, Long Beach, CA; and Division of Cardiology, ${ }^{\mathrm{b}}$ University of California, Irvine, CA.

Reprint requests: Gregory S. Thomas, MD, MPH, FACC, FASNC, MemorialCare Heart \& Vascular Center, Long Beach Memorial Medical Center and Miller Children's Hospital of Long Beach, 2801 Atlantic Ave, Long Beach, CA 90806; gthomas1@memorialcare. org.

J Nucl Cardiol 2013;20:324-8.

$1071-3581 / \$ 34.00$

Copyright (C) 2013 American Society of Nuclear Cardiology.

doi:10.1007/s12350-013-9695-1 compromised due to lack of an appropriate heart rate being achieved. A proposed algorithm was described in our previous editorial on this topic. ${ }^{10} \mathrm{~A}$ randomized clinical trial of a novel protocol in which symptom limited exercise is performed and if an adequate heart rate or an ischemic endpoint is not achieved, the administration of regadenoson is delayed until three minutes post exercise during a five minute recovery period of slow walking (termed RegRecovery) is now underway. ${ }^{11}$

In the current issue of the Journal, Cabrera and colleagues present an excellent contribution to the literature with the third study evaluating hemodynamic and stress testing variables in patients undergoing RegLowEx myocardial perfusion imaging (MPI). ${ }^{12}$ In this retrospective analysis of 485 patients who underwent RegLowEx protocol for MPI compared with 887 patients who underwent supine regadenoson imaging, the authors found the RegLowEx protocol to be safe. The use of aminophylline to reverse adverse effects was significantly lower in the RegLowEx group than the regadenoson supine group, $5.6 \%$ vs $11.4 \%$, respectively. Understandably, in a retrospective analysis, the two study populations had different baseline characteristics. In their sample, subjects undergoing low-level exercise were significantly younger and had fewer comorbidities. Although resting heart rates were similar in the two groups (69 bpm in regadenoson group, $67 \mathrm{bpm}$ in RegLowEx), as would be expected there was a more prominent increase in the RegLowEx group, 103 vs $83 \mathrm{bpm}$, respectively. On average, systolic blood pressure decreased by $9 \mathrm{~mm} \mathrm{Hg}$ in the regadenoson only group but increased by $5 \mathrm{~mm} \mathrm{Hg}$ in the low-level exercise group. Summed difference score on MPI was not significantly different although this comparison must be viewed with caution given the baseline differences between the study groups.

The rate of common symptoms including headache, chest pain, and dyspnea was similar in both groups. Flushing and abdominal discomfort were more common in the regadenoson only group whereas dizziness and fatigue were more common in the RegLowEx group. The RegLowEx group included patients with left bundle branch block (LBBB). Similar to previous two studies, 
the hemodynamic changes were not concerning in the low-level exercise group. The benefits of improved imaging characteristics have been well-documented not just with regadenoson but for other vasodilators. The primary reason for this is alterations in blood flow that reduce liver and gut uptake and possibly improved detection of ischemia.

An implication of this and previous studies is: should RegLowEx protocol be applied to all patients undergoing regadenoson MPI who are able to walk? To be conservative, at least the same absolute contraindications to symptom-limited exercise should be applied to RegLowEx (Table 1). Specifically, this includes acute myocardial infarction within 2 days, unstable angina, uncontrolled symptomatic cardiac arrhythmias, symptomatic severe aortic stenosis, decompensated heart failure, acute pulmonary embolism, acute aortic dissection, and acute myocarditis/pericarditis. ${ }^{13}$ Furthermore, RegLowEx should not include patients with LBBB or right ventricular pacing. As noted in this study and others, the heart rate increase in RegLowEx is not insignificant. In this study, mean heart rate increased by $34 \mathrm{bpm}$. For some patients this may nearly achieve $85 \%$ of predicted heart rate using the 220-age formula. For example, using 220-age, an 80-year-old patient's $85 \%$ of the predicted heart rate of $119 \mathrm{bpm}$, is within the standard deviation of the results of this study. Although the current study included patients with LBBB, we suggest excluding such patients from exercise as the increase in heart rate even with low-level exercise has the potential to produce false positive MPI results. ${ }^{14}$

If a conservative approach for patient selection is chosen, the next logical question is: why not attempt symptom-limited exercise in all patients who are being considered candidates for RegLowEx? The addition of symptom-limited stress test would further aid in prognosis. Indeed, when comparing patients randomized to RegPeakEx vs dipyridamole with exercise, Parker and colleagues found that $50 \%$ of patients undergoing symptom-limited exercise in the RegPeakEx group were able to achieve at least $85 \%$ predicted heart rate and therefore did not require regadenoson at all. However, they randomized only those capable of walking on a treadmill, overestimating the number of overall patients referred for vasodilator stress who could achieve $85 \%$ of predicted heart rate. This would suggest that, it may be prudent to attempt symptom-limited exercise in all patients who are considered candidates for low-level exercise while following the ACC/AHA guidelines for exercise stress testing (Table 1).

If a decision is made for symptom-limited exercise, what protocol should be followed for those who are unable to achieve $85 \%$ of predicted heart rate? This question has yet to be conclusively answered. Options include RegPeakEx/RegNearPeakEx/RegPostPeakEx, during low-level exercise in mid recovery from symptom-limited exercise (RegRecovery) or once patient has returned to baseline (termed RegLateRecovery).

Table 1. Absolute and relative contraindications for exercise stress testing (Adapted from ACC/AHA guidelines for stress testing $2002^{13}$ )

\author{
Absolute contraindications \\ Acute myocardial infarction within 2 days \\ High-risk unstable angina \\ Uncontrolled cardiac arrhythmias causing symptoms or hemodynamic compromise \\ Symptomatic severe aortic stenosis \\ Uncontrolled symptomatic heart failure \\ Acute pulmonary embolus or pulmonary infarction \\ Acute myocarditis or pericarditis \\ Acute aortic dissection \\ Relative contraindications \\ Left main stenosis \\ Moderate stenotic valvular heart disease \\ Electrolyte abnormalities \\ Severe arterial hypertension \\ Tachyarrhythmias or bradyarrhythmias \\ Hypertrophic cardiomyopathy and other forms of outflow tract obstruction \\ Mental or physical impairment leading to inability to exercise adequately \\ High-degree atrioventricular block
}







Each has potential advantages and disadvantages (Table 2).

The ideal stress test would not only provide excellent sensitivity and specificity for ischemia and prognosis but should also be very safe. Given the frequency at which stress tests are ordered, even low event rates of major complications are unacceptable. Theoretically, the safest tests employing regadenoson would be expected to be supine test followed by RegLowEx and then RegLateRecovery. The RegPeakEx protocol could eliminate regadenoson usage in a substantial percent of patients scheduled for vasodilator MPI and would provide further prognostic information. However, for those patients who undergo symptom-limited exercise but still require regadenoson, administering it at peak or during recovery can produce a physiologic milieu of "double stress." In the subset of patients who may have ischemic electrocardiographic changes or ischemic pain during exercise or recovery, administration of regadenoson with RegPeakEx or RegRecovery protocols could lead to excessive ischemia. A protocol of RegLateRecovery eliminates the theoretical concern for "double stress" but prolongs the study or requires two separate stress tests. As these techniques are novel, any protocol with symptom-limited exercise should be evaluated in studies of larger sample sizes in the form of randomized controlled trials or potentially a registry, which would allow its evaluation in a large number of subjects.

Test supervision and clinician competence is of utmost importance in performing protocols using symptom-limited exercise with regadenoson. In these more complex protocols, a clinician must be able to quickly evaluate ischemic symptoms and electrocardiographic changes to make a rapid decision whether to administer regadenoson if goal heart rate is not achieved. The radiotracer should be administered if an ischemic endpoint is achieved regardless of heart rate. In protocols in which patients receive regadenoson in recovery, regadenoson should not be administered if patients reach an ischemic endpoint during recovery to avoid the potential of "double stress." Such patients would be expected to be more safely evaluated with coronary angiography, regadenoson only testing or potentially with RegLowEx. Laboratories using symptom-limited exercise with regadenoson should carefully consider the ability of the supervising clinician to evaluate ischemia on the fly.

Pending ongoing and anticipated studies of regadenoson with symptom-limited exercise, the RegLowEx protocol, while not providing the additional prognostic information of maximal exercise testing, remains a viable and theoretically the safest option combining regadenoson with exercise while improving image quality and increasing the tolerability of regadenoson MPI.

\section{Disclosures}

GST serves as a consultant, speaker, and on a research steering committee for Astellas Pharma Global Development. He has received research grants from PGX Health and Astellas Pharma Global Development.

\section{References}

1. Henzlova MJ, Cerqueira MD, Hansen CL, Taillefer R, Yao S-S. Stress protocols and tracers. J Nucl Cardiol 2009;16:331.

2. Hendel RC, Frost L. Off-label, but on-target: Use of regadenoson with exercise. J Nucl Cardiol 2012. doi:10.1007/s12350-0129641-7.

3. Ghimire G, Hage FG, Heo J, Iskandrian AE. Regadenoson: A focused update. J Nucl Cardiol 2012. doi:10.1007/s12350-0129661-3.

4. Thomas GS, Thompson RC, Miyamoto MI, Ip TK, Rice DL, Milikien D, et al. The RegEx trial: A randomized, double-blind, placebo- and active-controlled pilot study combining regadenoson, a selective $\mathrm{A}(2 \mathrm{~A})$ adenosine agonist, with low-level exercise, in patients undergoing myocardial perfusion imaging. J Nucl Cardiol 2009;16:63-72.

5. Kwon DH, Cerqueira MD, Young R, Houghtaling P, Lieber E, Menon $\mathrm{V}$, et al. Lessons from regadenoson and low-level treadmill/regadenoson myocardial perfusion imaging: Initial clinical experience in 1263 patients. J Nucl Cardiol 2010;17:853-7.

6. Parker MW, Morales DC, Slim HB, Ahlberg AW, Katten DM, Cyr $\mathrm{G}$, et al. A strategy of symptom-limited exercise with regadenoson-as-needed for stress myocardial perfusion imaging: A randomized controlled trial. J Nucl Cardiol 2012. doi:10.1007/ s12350-012-9641-7.

7. Partington SL, Lanka V, Hainer J, Blankstein R, Skali H, Forman $\mathrm{DE}$, et al. Safety and feasibility of regadenoson use for suboptimal heart rate response during symptom-limited standard Bruce exercise stress test. J Nucl Cardiol 2012;19:970-8.

8. Aljaroudi WA, Alraies MC, Cerquiera MD, Jaber WA. Safety and tolerability of regadenoson in 514 SPECT MPI patients with and without coronary artery disease and submaximal exercise heart rate response. Eur J Nucl Med Mol Imaging 2013. doi:10.1007/ s00259-123-2296-4.

9. Thompson RC, Patil H, Thompson EC, Thomas GS, Al-Amoodi $\mathrm{M}$, Kennedy $\mathrm{KF}$, et al. Regadenoson pharmacologic stress for myocardial perfusion imaging: A three-way comparison between regadenoson administered at peak exercise, during walk recovery, or no-exercise. J Nucl Cardiol 2012. doi:10.1007/s12350-0129660-4.

10. Thomas GS, Hundal HS, Ellestad MH. Advanced hybrid stress testing: A potential new paradigm combining exercise and pharmacologic stress. J Nucl Cardiol 2012;19:887-90.

11. A study to assess regadenoson administration following an inadequate exercise stress test as compared to regadenoson alone for myocardial perfusion imaging (MPI) using single photon emission computed tomography (SPECT) (EXERRT). http://www.clinical trials.gov/ct2/show/NCT01618669?term=regadenoson $\&$ rank=1 accessed. Accessed 2013 Feb 5

12. Cabrera R, Husain Z, Palani G, Karthikeyan A, Dhanalakota S, Peterson E, et al. Comparison of hemodynamic and stress testing 
variables in patients undergoing regadenoson stress myocardial perfusion imaging to regadenoson with adjunctive low level exercise myocardial perfusion imaging. J Nucl Cardiol 2013 (submitted)

13. Gibbons RJ, Balady GJ, Bricker JT, Chaitman BR, Fletcher GF, Froelicher VF, et al. ACC/AHA 2002 guideline update for exercise testing: Summary article. A report of the American College of Cardiology/American Heart Association Task Force on Practice Guidelines (Committee to Update the 1997 Exercise Testing Guidelines). J Am Coll Cardiol 2002;40:1531-40.

14. Klocke FJ, Baird MG, Lorell BH, Bateman TM, Messer JV, Berman DS, et al. ACC/AHA/ASNC guidelines for the clinical use of cardiac radionuclide imaging-executive summary: A report of the American College of Cardiology/American Heart Association Task Force on Practice Guidelines (ACC/AHA/ASNC Committee to Revise the 1995 Guidelines for the Clinical Use of Cardiac Radionuclide Imaging). Circulation 2003;108:1404-18. 